\title{
考虑阻尼衰减的点位操作时间最优运动规划"
}

\author{
白有盾 陈 新 杨志军 \\ (广东工业大学广东省微纳加工技术与装备重点实验室 广州 510006)
}

\begin{abstract}
摘要: 阻尼在机械系统中普遍存在, 然而现有运动规划方法普遍不考虑阻尼衰减影响, 不能体现运动过程的振动衰减效应。 提出一种考虑阻尼衰减的时间最优运动规划模型。在优化模型中的定位误差约束条件基于采用 Laplace 方法获取的阻尼柔性 运动系统的残余振动响应进行定义。同时，定义了一种包含 7 个独立设计变量的三阶非对称 $\mathrm{S}$ 曲线运动规划模型以便于在运 动规划中充分考虑阻尼的影响。数值试验表明阻尼衰减效应对小阻尼运动系统的残余振动响应也有可能产生较大的影响。数 值试验与实际运动平台测试结果均表明: 优化后的运动规划中加速阶段的急动度要大于减速阶段, 以便充分利用阻尼衰减效 应来抑制残余振动，从而导致所得时间最优运动规划曲线为一种高加速低减速的非对称运动规划曲线。试验测试结果表明基 于所述时间最优运动规划模型得到的最优运动规划较之传统的对称 $\mathrm{S}$ 曲线运动规划方法, 可以显著地缩短满足定位精度要求 所需的时间。
\end{abstract}

关键词：非对称 S 曲线; 运动规划; 残余振动; 时间最优; 阻尼衰减

中图分类号: TG156

\section{Time-optimal Motion Profile Planning Considering Damping Attenuation for Point to Point Operation}

\author{
BAI Youdun CHEN Xin YANG Zhijun \\ (Guangdong Provincial Key Laboratory of Micro-Nano Manufacturing Technology and Equipment, \\ Guangdong University of Technology, Guangzhou 510006)
}

\begin{abstract}
The damping is existed in mechanical system, however, most of current motion planning approaches do not consider the damping attenuation effect, thus the real response of vibration during motion control can not be simulated. The time-optimal motion planning model considering damping is proposed. The related positioning error constraint of the presented optimization model is constructed based on the residual vibration response of damped flexible motion system obtained by using Laplace method. Also, a 3rd order asymmetric S-curve motion profile with 7 independent variables is proposed to consider the damping attenuation in motion profile design. Numerical simulation indicates that the damping may have the large influence on residual vibration response of lightly damped flexible motion system. Furthermore, both numerical simulation and experiment results reveal that the jerks' magnitude during acceleration section of the optimized motion profile are larger than those during deceleration section in order to make full use of damping attenuation to limit residual vibration, which results that the obtained time-optimal motion profile is the asymmetric profile with high acceleration and low deceleration. The experiment results indicate that the optimized motion profile obtained by the proposed time-optimal motion planning model can significantly reduce the required positioning time meeting the positioning accuracy demand.
\end{abstract}

Key words: asymmetric S-curve profile; motion planning; residual vibration; time-optimal; damping attenuation

0 前言

在半导体制造等精密工程领域中，如何在保持

* 国家自然科学基金(91648108, U1601202, 51675106)、广东省自然科学基 金(2015A030312008, 2015A030308004，2016A030308016)、广东科技计 划(2015B010104006, 2015B010104008, 2015B010133005)、国家重点研 发计划(2017YFF0105902)和广州科技规划(201510010281)资助项目。 20171222 收到初稿, 20180828 收到修改稿
定位精度的前提下尽可能地缩短定位时间对于提高 设备的生产效率和改善加工质量至关重要 ${ }^{[1]}$ 。解决 上述问题的关键技术之一为高速高精度运动规划。

通常而言，采用如二阶梯形速度运动规划曲线 可以极大地缩短运动规划时间。但上述高速运动规 划曲线存在速度突变, 容易激发较大的残余振动, 进而导致运动系统需要较多的振动衰减时间来满足 定位精度。由于速度规划中不存在突变, $\mathrm{S}$ 曲线运 
动规划被认为可以实现高速度运动且不会导致过大 残余振动。目前常见的 $\mathrm{S}$ 曲线运动规划主要有: 高 阶多项式 $S$ 曲线 ${ }^{[2]}$ 、正弦 $S$ 曲线 ${ }^{[1]}$ 、无限阶光滑 $S$ 曲线 ${ }^{[3]}$ 、非对称 $S$ 曲线 ${ }^{[4-7]}$ (AS-curve) 等。运动规划 的参数选取目前主要有以下两种方式: 基于规划曲 线几何光顺性和基于动力学响应分析方法。其中, 前者主要集中于设计满足电机最大加速度、最大速 度等物理性能指标下的运动规划曲线参数, 国内外 已经有大量文献对上述问题进行了研究 ${ }^{[3,8-11]}$ 。后者 是通过对不同运动规划参数在柔性运动系统中的动 力学响应来确定最佳运动规划参数。MECKL ${ }^{[12]}$ 利 用频率法分析了 $\mathrm{S}$ 曲线运动规划中加减速时间参数 对小阻尼运动系统响应的影响, 并以最小化残余振 动能量为目标对 $\mathrm{S}$ 曲线运动参数进行了优化。杨志 军等 ${ }^{[13]}$ 利用 ADAMS 联合仿真优化方式获得了粘片 机焊头机构在满足电机能力与残余振动约束下的时 间最短运动速度规划参数。韩国 HA 等 ${ }^{[14-16]}$ 利用 Laplace 频率分析方法研究了非对称 S 曲线(AS-curve) 在小阻尼运动系统的振动响应, 并利用零极点配置 方法分别对长行程与短行程工况下的非对称 $\mathrm{S}$ 曲线 运动参数进行了优化整定。在工程实践中, 数字滤 波输入整形技术 ${ }^{[17-19]}$ 也可以被认为是一种基于运动 机构动力学特性分析来有效抑制运动系统残余振动 的方法。

由于实际运动系统都带有一定的柔性, 基于运 动机构动力学响应的运动规划参数优化方法普遍要 优于单纯仅考虑物理约束条件的基于规划曲线光顺 性的运动规划参数选取方法。然而, 现有的基于运 动机构动力学响应的运动规划参数优化方法普遍仅 仅考虑运动机构固有频率对振动响应的影响, 通常 都假定机构的阻尼小到可以忽略来避免讨论阻尼对 机构响应的影响。而实际上, 根据动力学理论可知 阻尼会影响机构振动响应的相位和振幅, 进而影响 残余振动响应及定位精度。

本文采用 Laplace 方法构建了运动机构的固有频 率、阻尼、运动规划参数等与运动机构残余振动响 应之间的函数关系, 并进而建立了考虑阻尼影响的 定位精度约束条件。上述定位约束条件与最大急动 度、最大加速度等物理约束条件共同构成了时间最 优运动规划模型中的约束条件。本文所提的时间最 优运动规划模型充分考虑阻尼对残余振动的影响, 为高速高精密运动规划提供了一种通用优化方法。

\section{1 运动规划曲线定义}

考虑到运动规划曲线在运动控制卡中的实现便
利性, 本文选取三阶多项式 $\mathrm{S}$ 曲线作为时间最优运 动规划模型中的待优化运动规划曲线。由于常规的 对称 $\mathrm{S}$ 曲线运动规划可以被视为非对称 $\mathrm{S}$ 曲线运动 规划中的一个特例, 本文中的运动规划采用三阶多 项式非对称 $\mathrm{S}$ 曲线。为进一步增加优化模型的设计 自由度(增加更多的独立设计变量), 本文对现有的 非对称 S 曲线运动规划 ${ }^{[4-7]}$ 进行了拓展定义。

本文中所采用的非对称 $\mathrm{S}$ 曲线的急动度定义 如下

$$
\operatorname{Jerk}(t)=\left\{\begin{array}{l}
J_{1}\left(0<t \leqslant t_{1}\right) \\
-J_{2}\left(t_{2}<t \leqslant t_{3}\right) \\
-J_{3}\left(t_{4}<t \leqslant t_{5}\right) \\
J_{4}\left(t_{6}<t \leqslant t_{7}\right) \\
0(\text { 其他 })
\end{array}\right.
$$

式中, $J_{i}$ 为急动度幅值, $t_{i}$ 为运动规划曲线的时间 参数。为便于后续公式推导, 上述急动度 $J_{i}$ 均设定 为正值。此外, 定义了另一组等价的运动规划参数 $T_{i}=t_{i}-t_{i-1}(i=1,2, \cdots, 7)$ 用于简化后续公式表述。

$\mathrm{S}$ 曲线运动规划需要满足如下三个基本约束条 件: (1) 匀速段中的加速度为 0 ; (2) 运动终止时刻 的加速度为 0 ; (3) 运动终止时刻的速度为 0 。利用 式(1), 以上三约束条件可以表示为

$$
\left\{\begin{array}{l}
J_{1} T_{1}-J_{2} T_{3}=0, \quad J_{3} T_{5}-J_{4} T_{7}=0 \\
J_{1} T_{1}\left(T_{1}+2 T_{2}+T_{3}\right)-J_{3} T_{5}\left(T_{5}+2 T_{6}+T_{7}\right)=0
\end{array}\right.
$$

对式(1)进行三次积分, 可以获得 $\mathrm{S}$ 曲线运动规 划的终止时刻位移为

$$
\begin{aligned}
D= & \frac{1}{4} J_{1} T_{1}\left(T_{1}+2 T_{2}+T_{3}\right)\left(2 T_{4}+2 T_{5}+2 T_{6}+T_{3}+T_{7}\right)+ \\
& \frac{1}{24} J_{1} T_{1}\left(T_{1}^{2}-T_{3}^{2}\right)-\frac{1}{24} J_{3} T_{5}\left(T_{5}^{2}-T_{7}^{2}\right)=Q
\end{aligned}
$$

式中, $Q$ 为运动规划位移。

联立求解式(2)与(3), 可得

$$
\begin{gathered}
J_{i}=\frac{24 Q}{L} \times \frac{\left|G_{i}\right|}{T_{2 i-1}} \quad(i=1,2,3,4) \\
G_{1}=-G_{2}=T_{5}+2 T_{6}+T_{7} \\
G_{3}=-G_{4}=-\left(T_{1}+2 T_{2}+T_{3}\right) \\
L=3\left(T_{1}+2 T_{2}+T_{3}\right)\left(T_{5}+2 T_{6}+T_{7}\right) \times \\
\left(T_{1}+2 T_{2}+3 T_{3}+4 T_{4}+3 T_{5}+2 T_{6}+T_{7}\right)+ \\
\left(T_{5}+2 T_{6}+T_{7}\right)\left(T_{1}^{2}-T_{3}^{2}\right)-\left(T_{1}+2 T_{2}+T_{3}\right)\left(T_{5}^{2}-T_{7}^{2}\right)
\end{gathered}
$$

利用式(1)与(4), 本文所采用的运动规划曲线的 加速度、速度、位移等规划曲线可以定义为

$$
\begin{aligned}
& \operatorname{Acc}(t)=\frac{24 Q}{L} \sum_{i=1}^{4} \frac{G_{i}}{T_{2 i-1}}\left(S_{2 i-2}-S_{2 i-1}\right) \\
& \operatorname{Vel}(t)=\frac{12 Q}{L} \sum_{i=1}^{4} \frac{G_{i}}{T_{2 i-1}}\left(S_{2 i-2}^{2}-S_{2 i-1}^{2}\right)
\end{aligned}
$$




$$
\operatorname{Dis}(t)=\frac{4 Q}{L} \sum_{i=1}^{4} \frac{G_{i}}{T_{2 i-1}}\left(S_{2 i-2}^{3}-S_{2 i-1}^{3}\right)
$$

$S_{i}=\left(t-t_{i}\right) \times \operatorname{Heaviside}\left(t-t_{i}\right) \quad i=0,1, \cdots, 7 \quad t_{0}=0$

根据式(1)、(5)、(6)、(7), 本文所定义的非对 称 $\mathrm{S}$ 曲线运动规划的急动度、加速度、速度、位移 规划曲线绘制在图 1 中。

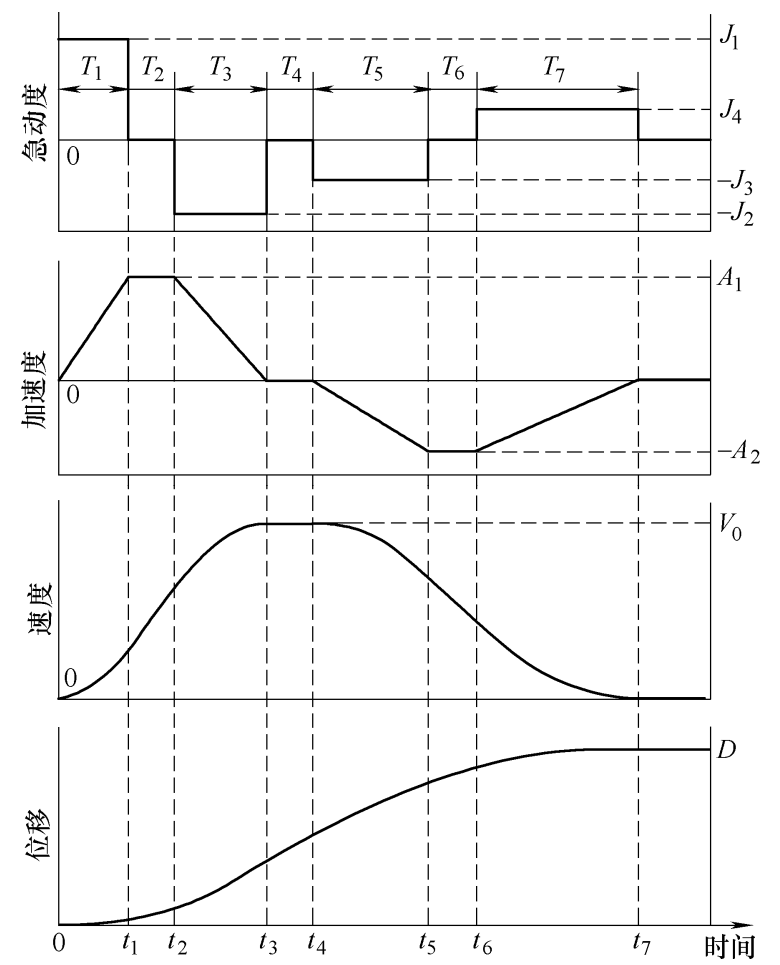

图 1 非对称 $\mathrm{S}$ 运动规划曲线示意图

2 时间最优运动规划模型

\section{1 运动规划的物理约束条件}

如图 1 所示, 运动规划的最大加速度出现在 $\left[t_{1}, t_{2}\right]$ 或 $\left[t_{5}, t_{6}\right]$, 最大速度出现在 $\left[t_{3}, t_{4}\right]$ 。因此, 非 对称 $\mathrm{S}$ 曲线运动规划的物理约束条件可以表示为

$$
\left\{\begin{array}{l}
0<J_{1}, J_{2}, J_{3}, J_{4} \leqslant J_{m} \\
A_{1}=J_{1} T_{1} \leqslant A_{m}, A_{2}=J_{3} T_{5} \leqslant A_{m} \\
V_{0}=J_{1} T_{1}\left(T_{1}+2 T_{2}+T_{3}\right) / 2 \leqslant V_{m}
\end{array}\right.
$$

式中, $J_{m}, A_{m}, V_{m}$ 分别为许用最大急动度、许用最大 加速度、许用最大速度。

将式(4)代入式(8), 物理约束条件可以表示为

$$
\begin{cases}\frac{24 Q}{J_{m} L} \times \frac{\left|G_{i}\right|}{T_{2 i-1}}-1 \leqslant 0 & i=1,2,3,4 \\ \frac{24 Q}{A_{m} L} \times\left|G_{1}\right|-1 \leqslant 0 & \frac{24 Q}{A_{m} L} \times\left|G_{3}\right|-1 \leqslant 0 \\ \frac{12 Q}{V_{m} L} \times\left|G_{1} G_{3}\right|-1 \leqslant 0 & \end{cases}
$$

\section{2 残余振动定位误差约束条件}

欠阻尼柔性运动系统通常可以用图 2 所示的双 质量块运动系统描述。其中, $x$ 为质量块 $M$ 的绝对 位置, $y$ 为质量块 $m$ 相对于质量块 $M$ 的振动位移, $k$ 为运动系统的刚度, $c$ 为运动系统的阻尼。

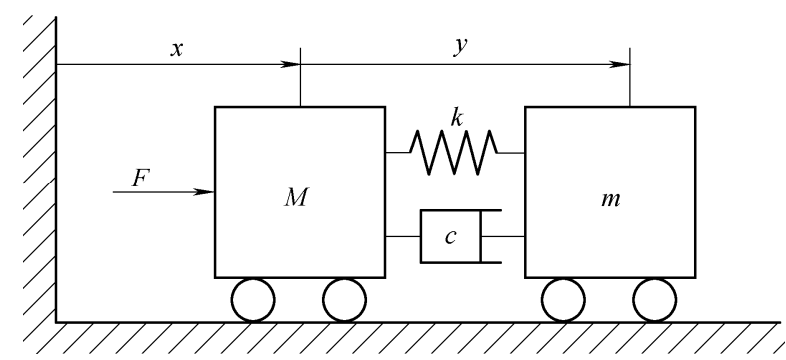

图 2 双质量块运动系统示意图

图 2 所示的双质量块运动系统的动力学方程为

$$
m(\ddot{x}+\ddot{y})+c \dot{y}+k y=0
$$

由式(10)可以得到 $x$ 与 $y$ 之间的传递函数为

$$
\frac{Y(s)}{X(s)}=\frac{-s^{2}}{s^{2}+2 s \omega_{n} \xi+\omega_{n}^{2}}
$$

式中, $X(s)$ 与 $Y(s)$ 分别为 $x$ 与 $y$ 的 Laplace 频域表 达式, $\omega_{n}$ 与 $\xi$ 分别为欠阻尼运动系统的固有频率 $\left(\omega_{n}=\sqrt{k / m}\right)$ 与阻尼比 $(\xi=c / 2 \sqrt{m k})$ 。

假定质量块 $M$ 在驱动力 $F$ 的作用下按照上一节 定义的非对称 $\mathrm{S}$ 曲线运动规划运动, 则根据式(5)可 以求得质量块 $M$ 的加速度 Laplace 表达式为

$$
\begin{gathered}
s^{2} X(s)= \\
\frac{24 Q}{L} \times \frac{1}{s^{2}} \sum_{i=1}^{4} \frac{G_{i}}{T_{2 i-1}}\left(\exp \left(-s t_{2 i-2}\right)-\exp \left(-s t_{2 i-1}\right)\right)
\end{gathered}
$$

式中, $t_{0}=0$ 。

根据式(11)和(12), 可以利用 Laplace 逆变换获 得质量块 $m$ 在式(5)对应的非对称 $\mathrm{S}$ 曲线运动规划作 用下的位移响应为

$$
\begin{gathered}
y(t)= \\
\frac{24 Q}{L} \times\left(\frac{1}{\sqrt{1-\xi^{2}} \omega_{n}^{3}} \sum_{i=1}^{4} \frac{G_{i}}{T_{2 i-1}}\left(A_{2 i-1} H_{2 i-1}-A_{2 i-2} H_{2 i-2}\right)-\right. \\
\frac{2 \xi}{\omega_{n}^{3}} \sum_{i=1}^{4} \frac{G_{i}}{T_{2 i-1}}\left(H_{2 i-1}-H_{2 i-2}\right)+ \\
\left.\frac{1}{\omega_{n}^{2}} \sum_{i=1}^{4} \frac{G_{i}}{T_{2 i-1}}\left(\left(t-t_{2 i-1}\right) H_{2 i-1}+\left(t_{2 i-2}-t\right) H_{2 i-2}\right)\right) \\
A_{i}=\sin \left(\sqrt{1-\xi^{2}} \omega_{n}\left(t-t_{i}\right)+2 \phi\right) \exp \left(-\omega_{n} \xi\left(t-t_{i}\right)\right) \\
H_{i}=\text { Heaviside }\left(t-t_{i}\right) \quad \phi=\arccos (\xi) \\
i=0,1, \cdots, 7 \quad t_{0}=0
\end{gathered}
$$


对式(13)进行时间微分, 可得对应的速度响应 为

$$
\begin{gathered}
v(t)= \\
\frac{24 Q}{L} \times\left(\frac{-1}{\sqrt{1-\xi^{2}} \omega_{n}^{2}} \sum_{i=1}^{4} \frac{G_{i}}{T_{2 i-1}}\left(B_{2 i-1} H_{2 i-1}-B_{2 i-2} H_{2 i-2}\right)+\right. \\
\left.\frac{1}{\omega_{n}^{2}} \sum_{i=1}^{4} \frac{G_{i}}{T_{2 i-1}}\left(H_{2 i-1}-H_{2 i-2}\right)\right) \\
B_{i}=\sin \left(\sqrt{1-\xi^{2}} \omega_{n}\left(t-t_{i}\right)+\phi\right) \exp \left(-\omega_{n} \xi\left(t-t_{i}\right)\right)
\end{gathered}
$$

由于运动终止时刻 $t_{7}$ 后的残余振动响应与定位 精度直接相关, 因此可以采用时间偏移 $\hat{t}=t-t_{7}$ 来 获得运动终止时刻 $t_{7}$ 后的位移与速度响应为

$$
\begin{gathered}
y(\hat{t})=\frac{1}{\sqrt{1-\xi^{2}} \omega_{n}^{3}} \times \frac{24 Q}{L} \sum_{i=1}^{4} \frac{G_{i}}{T_{2 i-1}}\left(A_{2 i-1}^{\prime}-A_{2 i-2}^{\prime}\right) \\
v(\hat{t})=\frac{-1}{\sqrt{1-\xi^{2}} \omega_{n}^{2}} \times \frac{24 Q}{L} \sum_{i=1}^{4} \frac{G_{i}}{T_{2 i-1}}\left(B_{2 i-1}^{\prime}-B_{2 i-2}^{\prime}\right) \\
A_{i}^{\prime}=\sin \left(\sqrt{1-\xi^{2}} \omega_{n}\left(\hat{t}+t_{7}-t_{i}\right)+2 \phi\right) \exp \left(-\omega_{n} \xi\left(\hat{t}+t_{7}-t_{i}\right)\right) \\
B_{i}^{\prime}=\sin \left(\sqrt{1-\xi^{2}} \omega_{n}\left(\hat{t}+t_{7}-t_{i}\right)+\phi\right) \exp \left(-\omega_{n} \xi\left(\hat{t}+t_{7}-t_{i}\right)\right)
\end{gathered}
$$

由式(15)可以看出 $t_{7}$ 时刻后的残余振动响应为 4 个具有相同频率 $\sqrt{1-\xi^{2}} \omega_{n}$ 但不同振幅与相位的指 数衰减正弦振荡响应的叠加。其中, 各振动响应组 成部分的相位 $2 \phi$ 完全由系统阻尼比 $\xi$ 确定, 对应的 振幅与系统阻尼比直接相关。容易看出, 当系统阻 尼比 $\xi$ 不为零时, 式(15)中各残余振动响应分部的幅 值将存在差异。反之, 如果采用阻尼比可以忽略 $(\xi \approx 0)$ 的假设, 则式(15)中各残余振动响应分部的 幅值将完全相同。显然采用式(15)带有显式阻尼比 $\xi$ 的残余振动响应公式更符合实际运动系统响应。

根据作者在文献[20]所述的方法(详细推导过程 见第 2.4 节), 定位误差约束条件可以表示为

$$
\sqrt{y^{2}(\hat{t})+\left(v(\hat{t}) / \omega_{n}\right)^{2}} \leqslant \varepsilon
$$

式中, $\varepsilon$ 为许用最大定位误差。

将式(15)、(16)代入式(17), 定位误差约束条件 变为

$$
\sqrt{\frac{24 Q}{\sqrt{1-\xi^{2}} \omega_{n}^{3} L} \times} \sqrt{\left(\sum_{i=1}^{4} \frac{G_{i}}{T_{2 i-1}}\left(A_{2 i-1}^{\prime}-A_{2 i-2}^{\prime}\right)\right)^{2}+\left(\sum_{i=1}^{4} \frac{G_{i}}{T_{2 i-1}}\left(B_{2 i-1}^{\prime}-B_{2 i-2}^{\prime}\right)\right)^{2}} \leqslant \varepsilon
$$

\section{3 时间最优运动规划模型的目标函数}

满足定位精度要求的运动时间包含以下两部分
时间: 运动规划时间之和 $\sum_{i=1}^{7} T_{i}$ 与满足精度要求所需 的衰减时间 $\hat{t}$ 。因此, 时间最优运动规划模型的目 标函数可以表示为

$$
\min \sum_{i=1}^{7} T_{i}+\hat{t}
$$

物理约束条件(9)、定位约束条件(18)与优化目 标函数(19), 时间最优运动规划优化模型可以总结 为

$$
\begin{aligned}
& \text { Find } T_{i}, \hat{t}(i=1,2, \cdots, 7) \\
& \text { to } \min \sum_{i=1}^{7} T_{i}+\hat{t} \\
& \text { s.t. }\left\{\begin{array}{l}
\frac{24 Q}{J_{m} L} \times \frac{G_{i} \mid}{T_{2 i-1}}-1 \leqslant 0(i=1,2,3,4) \\
\frac{24 Q}{A_{m} L} \times\left|G_{1}\right|-1 \leqslant 0 \frac{24 Q}{A_{m} L} \times\left|G_{3}\right|-1 \leqslant 0 \\
\frac{12 Q}{V_{m} L} \times G_{1} G_{3} \mid-1 \leqslant 0 \\
\frac{24 Q}{\sqrt{1-\xi^{2}} \omega_{n}^{3} L} \times \\
\sqrt{\left[\left(\sum_{i=1}^{4} \frac{G_{i}}{T_{2 i-1}}\left(A_{2 i-1}^{\prime}-A_{2 i-2}^{\prime}\right)\right)^{2}+\left(\sum_{i=1}^{4} \frac{G_{i}}{T_{2 i-1}}\left(B_{2 i-1}^{\prime}-B_{2 i-2}^{\prime}\right)\right)^{2}\right]} \leqslant \varepsilon
\end{array}\right.
\end{aligned}
$$

本文中的最优解均通过 MATLAB $囚$ 全局优化工 具箱获取。

\section{4 文献[20]定位误差约束条件的详细推导过程}

运动系统在规划终止时刻 $t_{7}$ 之后的残余振动能 量可以表示为残余动能与残余势能之和, 即

$$
\begin{gathered}
E_{\text {total }}(t)=E_{\mathrm{P}}(t)+E_{\mathrm{K}}(t)= \\
\frac{1}{2} k y^{2}(t)+\frac{1}{2} m v^{2}(t)
\end{gathered}
$$

式(21)中的动能部分可以改写为

$$
\frac{1}{2} m v^{2}(t)=\frac{1}{2} k \frac{v^{2}(t)}{k / m}=\frac{1}{2} k \frac{v^{2}(t)}{\omega_{n}^{2}}
$$

将式(22)代入式(21), 残余振动能量可以表示为

$$
\begin{gathered}
E(t)=\frac{1}{2} k y^{2}(t)+\frac{1}{2} k \frac{v^{2}(t)}{\omega_{n}^{2}}= \\
\frac{1}{2} k\left(\sqrt{y^{2}(t)+v^{2}(t) / \omega_{n}^{2}}\right)^{2}
\end{gathered}
$$

式(23)中 $\sqrt{y^{2}(t)+v^{2}(t) / \omega_{n}^{2}}$ 具有长度量纲, 可以 视为残余振动位移响应 $y(t)$ 的近似包络线。 $y(t)$ 与 $\sqrt{y^{2}(t)+v^{2}(t) / \omega_{n}^{2}}$ 之间的关系如图 3 所示。 


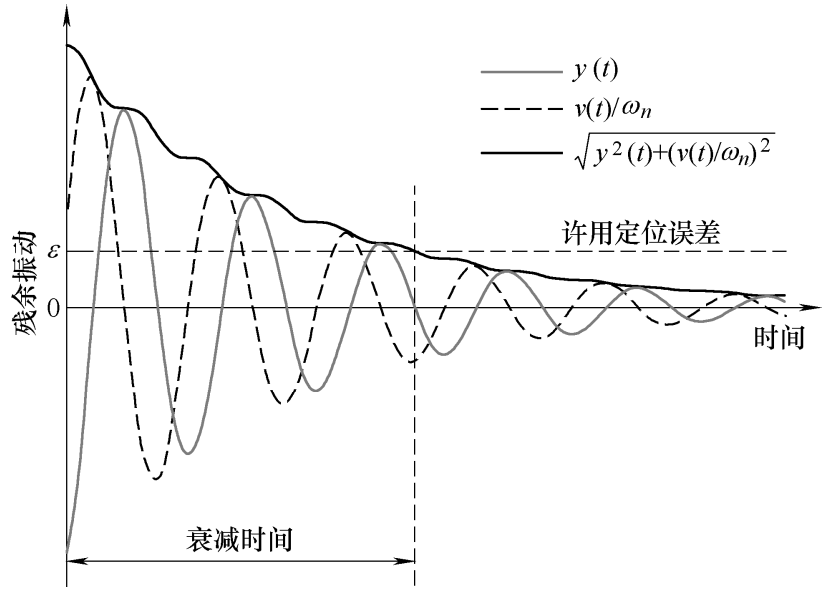

图 3 残余振动的位移响应与近似包络线的关系示意图

由图 3 容易看出, 位移响应近似包络线 $\sqrt{y^{2}(t)+v^{2}(t) / \omega_{n}^{2}}$ 为单调曲线。显然, 当残余振动 位移响应 $y(t)$ 满足许用定位误差要求时, 有

$$
\sqrt{y^{2}(t)+v^{2}(t) / \omega_{n}^{2}} \leqslant \varepsilon
$$

式(24)为优化模型(20)中所用的定位误差约束 条件。

\section{3 数值仿真}

为了验证阻尼比 $\xi$ 对式(20)所述时间最优运动 规划模型的影响, 采用如下数据进行数值仿真试验。

将表 1 所用数值仿真试验参数代入式(20)所述 的优化模型中进行求解。同时, 采用小阻尼假设, 令表 1 中阻尼比 $\xi=0$, 一并代入式(20)所述的优化 模型中进行求解。采用上述不同阻尼比所得的最优 运动规划参数列在表 2 中。

\section{表 1 数值仿真试验所用参数}

\begin{tabular}{cc}
\hline 参数 & 数值 \\
\hline 固有频率 $/ \mathrm{Hz}$ & 30 \\
阻尼比 & 0.05 \\
许用最大定位误差 $/ \mu \mathrm{m}$ & 5 \\
许用最大急动度 $/\left(\mathrm{m} / \mathrm{s}^{3}\right)$ & 5000 \\
许用最大加速度 $/\left(\mathrm{m} / \mathrm{s}^{2}\right)$ & 10 \\
许用最大速度 $/(\mathrm{m} / \mathrm{s})$ & 1 \\
运动行程 $/ \mathrm{mm}$ & 100 \\
\hline
\end{tabular}

表 2 不同阻尼比对应的最优运动规划参数 $(\mathrm{ms})$

\begin{tabular}{ccccccccc}
\hline$\xi$ & $T_{1}$ & $T_{2}$ & $T_{3}$ & $T_{4}$ & $T_{5}$ & $T_{6}$ & $T_{7}$ & $T_{\text {sum }}$ \\
\hline 0 & 21.7 & 82.2 & 2.0 & 10.0 & 2.0 & 82.2 & 21.7 & 221.6 \\
0.05 & 2.0 & 89.7 & 7.3 & 6.6 & 2.0 & 80.0 & 26.7 & 214.4 \\
\hline
\end{tabular}

由表 2 所列运动规划参数可以看出, 当采用阻 尼可以忽略 ( $\xi=0)$ 的假设时, $T_{1}=T_{7}, T_{2}=T_{6}$, $T_{3}=T_{5}$ 。较之于 $\xi=0.05$ 情形, $\xi=0$ 条件下所得非对 称 $\mathrm{S}$ 曲线运动规划参数仍然存在一定的对称性。这
主要是由于当不考虑阻尼衰减影响时, 系统输入激 励必须包含相互对称的加速与减速段，以便使所激 发的残余振动波形彼此相互抵消来最大程度抑制残 余振动。

将表 2 所列的运动规划参数代入式(13), 可以 得到含阻尼运动系统的振动位移响应, 如图 4 所 示。容易看出, 当不考虑阻尼衰减影响时, 振动位 移响应 $y(t)$ 在加速阶段 $\mathrm{A}$ 与减速阶段 $\mathrm{B}$ 基本对称; 而当考虑阻尼衰减影响时, 振动位移响应 $y(t)$ 在加 速阶段 $\mathrm{A}$ 的幅值要明显大于减速阶段 $\mathrm{B}$ 。上述结果 主要是因为加速阶段 $\mathrm{A}$ 的振动响应较之减速阶段 $\mathrm{B}$ 有更长的衰减时间, 在加速阶段采用更快速的运 动输入有利于充分缩短实际系统所需的定位时间。 此外, 如图 4 中运动系统的残余振动位移响应对比 可以看出: 是否考虑阻尼衰减效应可能对小阻尼运 动系统的振动响应有显著影响。显然, 考虑阻尼衰 减的最优运动规划参数能够更快速地完成高精度 定位运动。
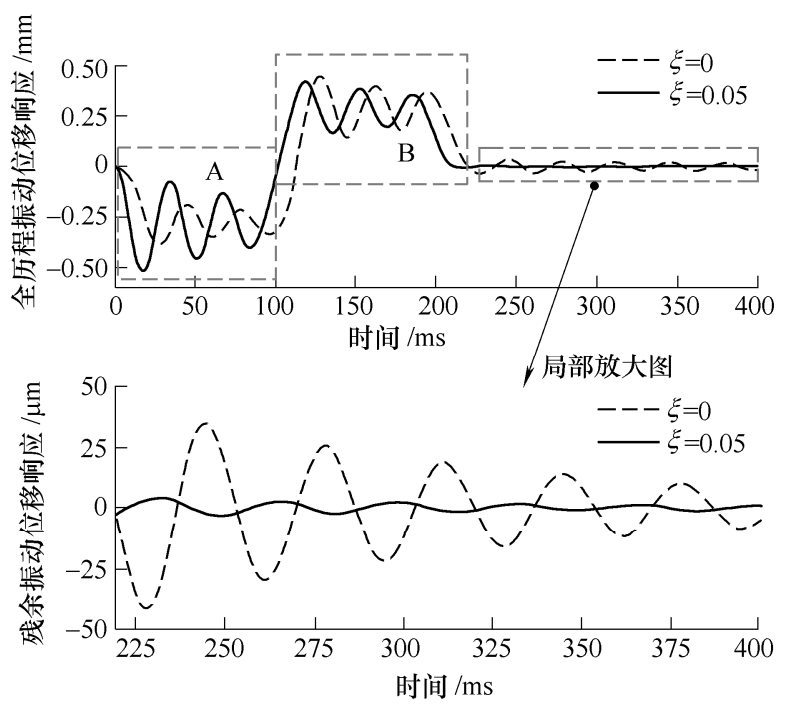

图 4 不同阻尼比对应的振动位移响应

\section{4 试验验证}

为了验证本文所述时间最优运动规划优化模型 的有效性，搭建了如图 5 所示的柔性悬臂梁运动测 试平台。

如图 5 所示，柔性悬臂梁固定在直线运动平台 上, 直线运动平台由无刷无铁芯直线电机(Akribis Inc)带动。柔性悬臂梁与直线运动平台共同构成了 图 2 所述的双质量块运动系统，悬臂梁相当于图 2 所示的质量块 $m$, 直线运动平台相当于图 2 所示的 质量块 $M$ 。上述无刷无铁芯直线电机由带光栅尺 (RENISHAW Inc.) 反馈的伺服驱动器驱动。一个 8 
轴运动控制卡(GOOGLTECH Inc.)被用于生成本文 所用的非对称 $\mathrm{S}$ 曲线运动规划指令。激光多普勒测 振仪(PSV-400-M2, Polytec Inc.)被用于测试柔性运 动平台的动力学参数与柔性悬臂梁末端的速度响 应, 作为系统激励输入的直线运动平台速度规划信 息通过光栅尺反馈信号获取。

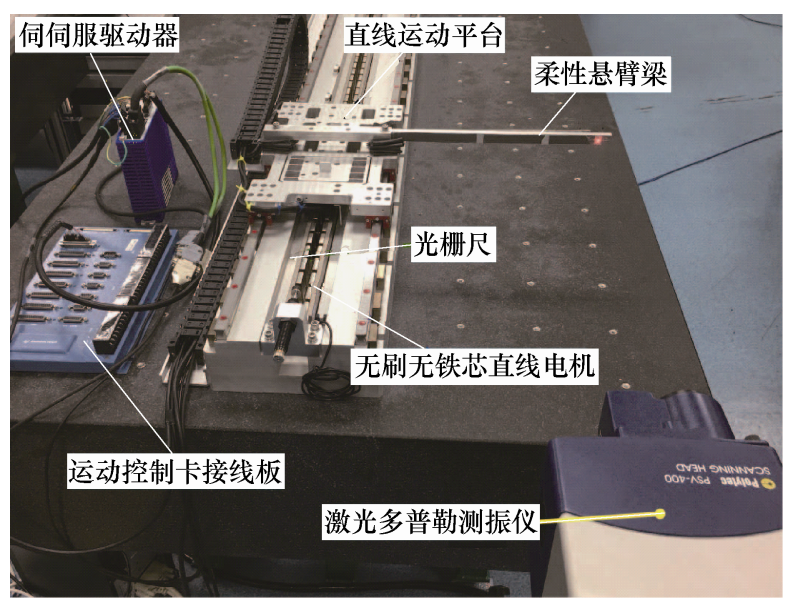

图 5 试验装置示意图

利用激光多普勒测振仪, 采用力锤敲击法获得 图 5 所示悬臂梁柔性运动系统的固有频率为 $\omega_{n}=50.38 \mathrm{~Hz}$, 并通过测振仪获取的时域衰减曲线 获取上述柔性运动平台的阻尼比为 $\xi=4.3 \%$ 。根据 直线电机的性能指标, 选取 $\varepsilon=15 \mu \mathrm{m}$ 作为许用最大 定位位置误差。根据动力学理论, 上述许用最大定 位误差 $\varepsilon=15 \mu \mathrm{m}$ 等价于许用定位速度误差 $V_{\text {error }}=\varepsilon \times \omega_{n} \approx 4.75 \mathrm{~mm} / \mathrm{s}$ 。柔性运动平台的动力学 参数与运动规划物理限制条件列在表 3 中。

表 3 试验装置平台参数表

\begin{tabular}{cc}
\hline 参数 & 数值 \\
\hline 固有频率 $/ \mathrm{Hz}$ & 50.38 \\
阻尼比 & 0.043 \\
许用最大定位位置误差 $/ \mu \mathrm{m}$ & 15 \\
许用最大定位速度误差 $/(\mathrm{mm} / \mathrm{s})$ & 4.75 \\
许用最大急动度 $/\left(\mathrm{m} / \mathrm{s}^{3}\right)$ & 10000 \\
许用最大加速度 $/\left(\mathrm{m} / \mathrm{s}^{2}\right)$ & 20 \\
许用最大速度 $/(\mathrm{m} / \mathrm{s})$ & 1 \\
\hline
\end{tabular}

利用表 3 所列物理限制条件分别采用文献[3]所 述方法和本文所述的优化模型获得相应的不考虑机 构动力学特性与考虑机构动力学特性的 $\mathrm{S}$ 曲线运动 规划参数。同时, 考虑到 $\mathrm{S}$ 曲线中匀速段存在与否, 本文对两组不同行程 $D=50 \mathrm{~mm}$ (短行程, 不存在匀 速段)和 $D=150 \mathrm{~mm}$ (长行程, 存在匀速段)进行了测 试。利用优化模型(20), 上述试验所用的运动规划 参数汇总在表 4 中。
表 4 运动规划参数 $(\mathrm{ms})$

\begin{tabular}{cccccc}
\hline & \multicolumn{2}{c}{$D=50 \mathrm{~mm}$} & & \multicolumn{2}{c}{$D=150 \mathrm{~mm}$} \\
\cline { 2 - 3 } \cline { 5 - 6 } & $\mathrm{A}$ & $\mathrm{B}$ & & $\mathrm{A}$ & $\mathrm{B}$ \\
\hline$T_{1}$ & 2.000 & 20.308 & & 2.000 & 22.501 \\
$T_{2}$ & 47.010 & 19.328 & & 48.000 & 28.732 \\
$T_{3}$ & 2.000 & 21.596 & & 2.000 & 20.035 \\
$T_{4}$ & 0.000 & 0.000 & & 98.000 & 71.545 \\
$T_{5}$ & 2.000 & 22.066 & & 2.000 & 20.261 \\
$T_{6}$ & 47.010 & 18.087 & & 48.000 & 45.965 \\
$T_{7}$ & 2.000 & 22.319 & & 2.000 & 20.647 \\
$T_{\text {sum }}$ & 102.020 & 123.703 & & 202.000 & 229.686 \\
\hline
\end{tabular}

注: $\mathrm{A}$ 组运动规划参数利用文献[3]所述方法获取; $\mathrm{B}$ 组运动规划参数 利用本文所述时间最优运动规划模型获取。

基于表 4 所述的运动规划参数, 利用前述实验 装置可以获得柔性悬臂梁运动平台的响应对比实验 结果, 如图 6 所示。除了由于运动惯量、摩擦力等 非线性因素导致的波动外, 图 6 所示的直线运动平 台的速度响应与表 4 所述的理想运动规划基本相同。 如图 6 所示, 柔性悬臂梁末端在采用文献[3]方法所 得运动规划曲线作用下的残余振动要远大于本文所 述优化模型所得的运动规划，对应的运动规划时间、 残余振动衰减时间及总定位时间的对比结果如表 5 所示。由表 4 与表 5 对比容易看出, 采用文献[3]方 法所得的运动规划时间总是短于采用本文所述时间 最优运动规划模型所得的运动规划时间, 但是文献 [3]方法所得的运动规划容易激发较大的残余振动, 从而导致其满足定位误差要求所需的残余振动衰 减时间要远大于本文所述的时间最优运动规划模 型。在 $D=50 \mathrm{~mm}$ (短行程, 不存在匀速段) 和 $D=150 \mathrm{~mm}$ (长行程, 存在匀速段)两种工作行程下, 采用本文优化模型获得运动规划对应的总定位时间 (运动规划时间+满足定位误差要求的振动衰减时间) 分别比采用文献[3]方法减少 $60.16 \%$ 和 $49.69 \%$ 。

表 5 运动定位时间对比(ms)

\begin{tabular}{ccccccc}
\hline & \multicolumn{2}{c}{$D=50 \mathrm{~mm}$} & & \multicolumn{2}{c}{$D=150 \mathrm{~mm}$} \\
\cline { 2 - 3 } \cline { 5 - 6 } & $\mathrm{A}$ & $\mathrm{B}$ & & $\mathrm{A}$ & $\mathrm{B}$ \\
\hline 运动规划时间 & 102 & 124 & & 202 & 229 \\
振动衰减时间 & 277 & 27 & & 375 & 11 \\
总定位时间 & 379 & 151 & & 477 & 240 \\
\hline$\left(t_{B}-t_{A}\right) / t_{A}(\%)^{*}$ & \multicolumn{3}{c}{-60.16} & & \multicolumn{2}{c}{-49.69} \\
\hline
\end{tabular}

注: ${ }^{*} t_{A}, t_{B}$ 分别为采用文献[3]方法与本文优化模型计算所得运动规 划曲线作用下柔性悬臂梁满足 $\varepsilon=15 \mu \mathrm{m}$ 所需的总定位时间。

对试验测试所得的悬臂梁末端速度响应进行快 速傅里叶分析, 可以得到两种行程工况下的频谱分 析对比图, 如图 7 所示。可以看出, 采用本文所述 的时间最优运动规划模型可以有效地避免激发运动 系统在其共振频率 $(50.38 \mathrm{~Hz})$ 处的振动响应。 

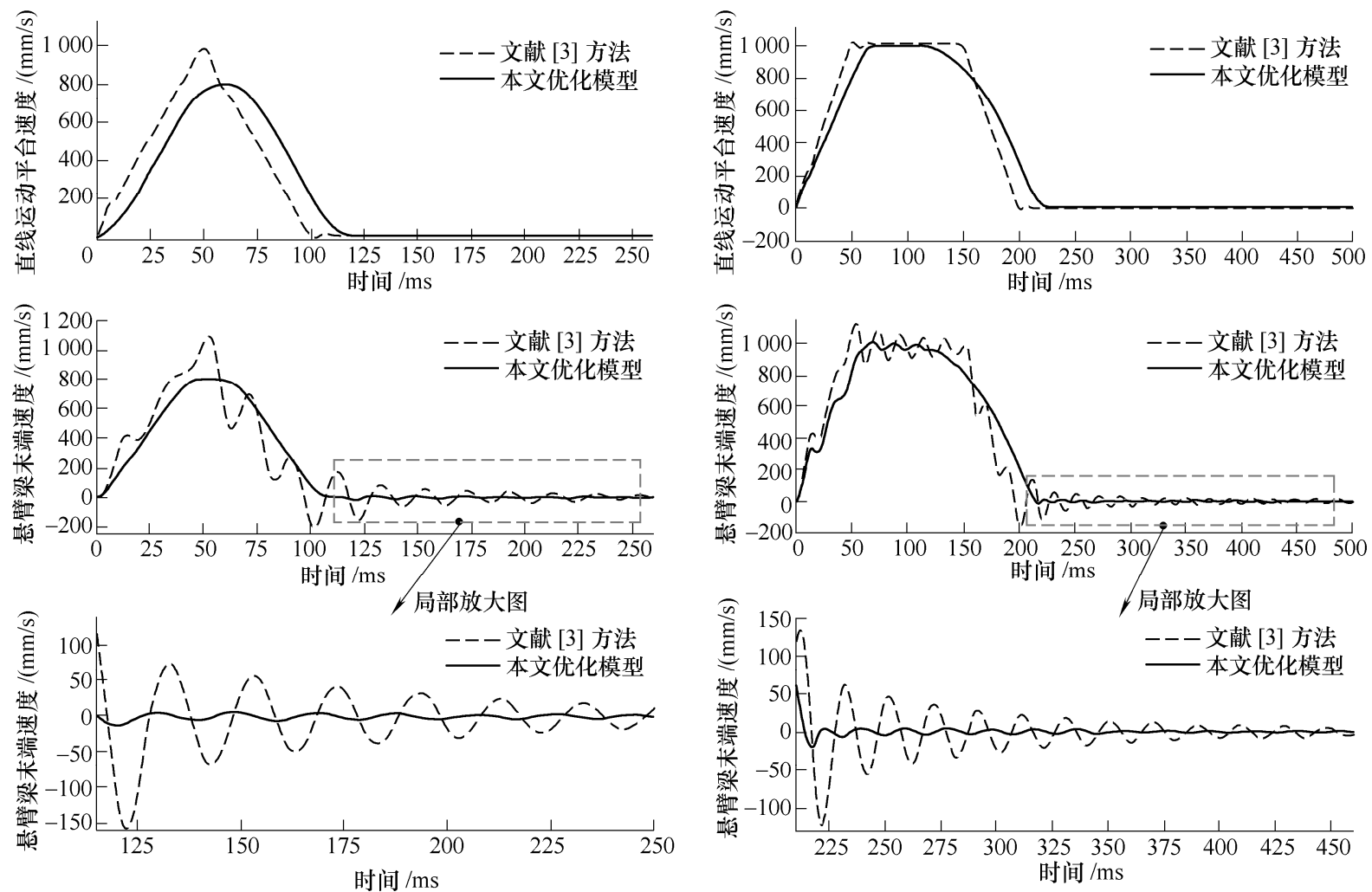

(a) $50 \mathrm{~mm}$ ( 短行程 )

(b) $150 \mathrm{~mm}$ ( 长行程 )

图 6 短行程与长行程工况下的悬臂梁柔性运动平台动力学响应对比

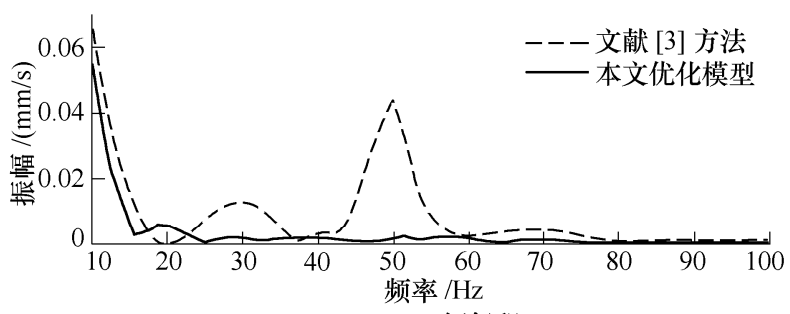

(a) $50 \mathrm{~mm}$ ( 短行程 )

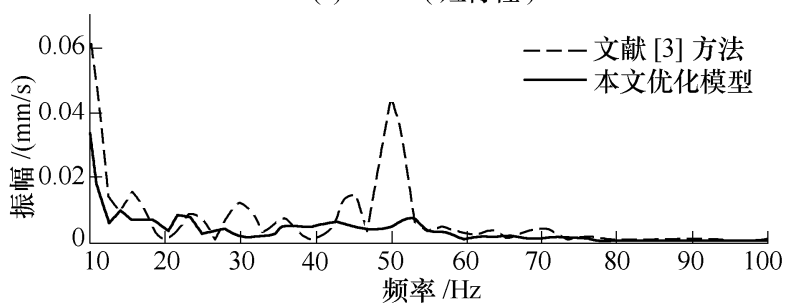

(b) $150 \mathrm{~mm}$ ( 长行程 )

图 7 悬臂梁末端速度响应 FFT 频谱图

此外, 将表 4 中采用本文优化模型所得的运动 规划参数代入式(4)中, 可以得到相应的各段急动度 幅值(表 6)。

表 6 时间最优 $S$ 运动规划曲线急动度 $\left(\mathrm{m} / \mathrm{s}^{3}\right)$

\begin{tabular}{ccccc}
\hline & $J_{1}$ & $J_{2}$ & $J_{3}$ & $J_{4}$ \\
\hline$D=50 \mathrm{~mm}$ & 988.18 & 923.55 & 910.11 & 896.94 \\
$D=150 \mathrm{~mm}$ & 888.87 & 998.26 & 743.09 & 729.20 \\
\hline
\end{tabular}

根据表 6 所列的不同行程下时间最优 $\mathrm{S}$ 运动规
划曲线的急动度数值, 可以看出: 两组运动规划参 数在加速阶段的急动度 $J_{1}$ 和 $J_{2}$ 要大于其减速阶段 的急动度 $J_{3}$ 和 $J_{4}$, 即优化后的运动规划曲线为典型 的高加速低减速非对称 $\mathrm{S}$ 曲线。上述结果主要是由 于运动系统存在的阻尼衰减效应所导致。由于急动 度 $J_{1}$ 和 $J_{2}$ 较之 $J_{3}$ 和 $J_{4}$ 有更长的实际衰减时间, 急 动度 $J_{1}$ 和 $J_{2}$ 对应的加速阶段采用更高的急动度有 利于提高运动速度; 同时, 急动度 $J_{3}$ 和 $J_{4}$ 对应的减 速阶段采用较小的急动度有利于避免激发较大的残 余振动。试验对比结果表明上述非对称 $\mathrm{S}$ 曲线运动 规划更有利于快速抑制残余振动。

\section{5 结论}

(1) 本文所用时间最优运动规划模型可以充分 考虑阻尼衰减对运动系统残余振动响应的影响。数 值试验结果表明是是否考虑阻尼衰减响应对小阻尼 运动系统的振动响应也可能产生较大的影响。数值 试验与实际运动装置测试结果均表明: 当考虑阻尼 衰减效应时，时间最优运动规划曲线为高加速低减 速的非对称 $\mathrm{S}$ 曲线运动规划。同时, 试验对比结果 也表明本文所述的非对称 $\mathrm{S}$ 曲线运动规划可以显著 地缩短满足定位精度要求所用的定位时间。 
(2) 本文拓展定义的非对称 S 曲线运动规划较 之现有文献中定义的非对称 S 曲线(AS-curve)增加 了两个独立设计变量, 有助于改善运动规划的优化 效果。同时, 本文也提供了上述非对称 $\mathrm{S}$ 曲线运动 规划的简洁解析公式, 便于在运动控制卡的编程使 用。

\section{参 考 文 献}

[1] LI Huaizhong, LE M D, GONG Z M, et al. Motion profile design to reduce residual vibration of high-speed positioning stages. IEEE/ASME Transactions on Mechatronics[J]. 2009, 14(2): 264-269.

[2] NGUYEN K D, NG T C, CHEN I M. On algorithms for planning s-curve motion profiles. International Journal of Advanced Robotic Systems[J]. 2008， 5(1): 99-106.

[3] LEE A Y, CHOI Y. Smooth trajectory planning methods using physical limits[J]. Journal of Mechanical Engineering Science, 2015, 229(12): 2127-2143.

[4] TSAY D M, LIN C F. Asymmetrical inputs for minimizing residual response[C]//EEE International Conference on Mechatronics, July 10-12, 2005, Taipei, China. IEEE, 2005: 235-240.

[5] REW K H, KIM K S. Using asymmetric s-curve profile for fast and vibrationless motion[C]//2007 International Conference on Control, Automation and Systems, October 17-20, 2007, Seoul, South Korea. IEEE, 2007: 500-504.

[6] REW K H, HA C W, KIM Y S. A practically efficient method for motion control based on asymmetric velocity profile[J]. International Journal of Machine Tools \& Manufacture, 2009, 49(7-8): 678-682.

[7] REW K H, KIM K S. A closed-form solution to asymmetric motion profile allowing acceleration manipulation[J]. IEEE Transactions on Industrial Electronics, 2010, 57(7): 2499-2506.

[8] 穆海华, 周云飞, 严思杰, 等. 超精密点对点运动 3 阶 轨迹规划算法研究[J]. 机械科学与技术, 2008, 27(2): 234-240.

MU Haihua, ZHOU Yunfei, YAN Sijie, et al. A third-order trajectory planning algorithm for ultra-precision point-to-point motion[J]. Mechanical Science and Technology for Aerospace Engineering. 2008, 27(2): 234-240.

[9] 杨玉龙. 高速主轴约束下的点位运动控制系统关键技 术研究[D]. 武汉：华中科技大学, 2014.

YANG Yulong. Study on technologies of point-to-point motion system under the constraint of high-speed
spindle[D]. Wuhan: Huazhong University of Science and Technology, 2014.

[10] SHI Xuguang, XU Bugong, XIE Wei, et al. Design and implementation of s-shape acceleration/deceleration algorithm based on rounding error compensation tactic.[C]//7th World Congress on Intelligent Control and Automation, June 25-27, 2008, Chongqing, China. IEEE, 2008: 7912-7916.

[11] 李纯军, 尹周平, 熊涛, 等. 一种点位运动快速轨迹规 划算法[J]. 组合机床与自动化加工技术，2011(3): 61-65.

LI Chunjun, YIN Zhouping, XIONG Tao, et al. A quick trajectory planning algorithm for point-to-point motion[J]. Modular Machine Tool \& Automatic Manufacturing Technique, 2011(3): 61-65.

[12] MECKL P H. Optimized s-curve motion profiles for minimum residual vibration[C]//American Control Conference, June 26-26, 1998, Philadelphia, USA. IEEE, 1998: 2627-2631.

[13] 杨志军, 曹占伦, 吴小洪, 等. 粘片机焊头机构快速启 停最优运动速度规划 [J]. 半导体技术, 2010，35(4): 373-377.

YANG Zhijun, CAO Zhanlun, WU Xiaohong, et al. Optimal velocity plan for die bonder considering positioning precision constraints[J]. Semiconductor Technology, 2010, 35(4): 373-377.

[14] HA C W, REW K H, KIM K S. Formulating a laplace domain approach for tuning motion profiles[C]//IEEE/ASME International Conference on Advanced Intelligent Mechatronics, July 3-7, 2011, Budapest, Hungary. IEEE, 2011: 356-360.

[15] HA C W, REW K H, KIM K S. Robust zero placement for motion control of lightly damped systems. IEEE Transactions on Industrial Electronics[J]. 2013 , 60(9): 3857-3864.

[16] HA C W, REW K H, KIM K S, et al. Tuning the s-curve motion profile in short distance case[C]//American Control Conference, June 17-19, 2013, Washington, DC，USA. IEEE， 2013： 4975-4980.

[17] SINGHOSE W. Command shaping for flexible systems: a review of the first 50 years. International Journal of Precision Engineering and Manufacturing[J]. 2009, 10(4): 153-168.

[18] SINGHOSE W, ELOUNDOU R, LAWRENCE J. Command generation for flexible systems by input shaping and command smoothing[J]. Journal of 
Guidance, Control, and Dynamics, 2010, 33(6) : 1697-1707.

[19] SINGHOSE W, VAUGHAN J. Reducing vibration by digital filtering and input shaping[J]. IEEE Transactions on Control Systems Technology, 2011, 19(6): 1410-1420.

[20] YANG Zhijun, BAI Youdun, CHEN Xin, et al. The residual vibration impact on the multiple target positioning time for array operation[C]//IEEE
International Conference on Information and Automation, August 1-3, 2016, Ningbo, China. IEEE, 2017: 50-55.

作者简介: 白有盾, 男, 1986 年出生, 博士。主要研究方向为运动控制 与宏微复合运动平台设计。

E-mail: youdun.bai@foxmail.com

杨志军(通信作者), 男, 1977 年出生, 博士, 教授。主要研究方向为机 电系统性能定制设计(动态性能定制和运动精度定制)、智能部件设计理 论与方法。

E-mail: yangzj@gdut.edu.cn 